\title{
Painfull Posterior Cruciate Ligament Ganglion Cyst. A Case Report
}

\author{
João Pinheiro, António Araújo and João Fonseca* \\ Department of Physical Medicine and Rehabilitation Coimbra Hospital and Universitary Centre, Portugal \\ *Corresponding author: João Fonseca, Department of Physical Medicine and Rehabilitation Coimbra Hospital and Universitary \\ Centre, Portugal
}

\section{Introduction}

Ganglion cysts (GC) are benign tumor-like lesions usually going out from mucinous degeneration of collagenous structures [1,2]. They could occur in several anatomic areas but GC arising from cruciate ligaments are rare $[3,4]$ with a prevalence of $0.36 \%$ or $0.8 \%$ respectively when diagnosed by magnetic resonance (MR) or by arthroscopy [3,5-6] However other studies of GC prevalence refer ranges from $0.2 \%$ to $1.9 \%$ [7-9], with posterior cruciate ligament ganglion cysts (PCLGC) being five times less frequent than those identify in anterior cruciate ligament $[5,10]$. This lesion is mainly diagnosed in people aged 20-40 years-old and a male predominance has been reported [5,11-13]. The etiology of PCLGC is not clear. They could appear from synovial herniation or congenital translocation of synovial cells. Mesenchymal stem cells proliferation with cysts formation or mucoid degeneration occurring in areas suffering chronic injuries are also reported [7,14-16]. Many of PCLGC are asymptomatic. When symptomatic the main clinical symptoms and signals includes knee pain and / or movement restrictions [17].
The knee joint could present a slight effusion, restriction to extension and particularly in extreme flexion $[1,4]$. The common classification of cruciate ligament cysts is supported on the position of the cyst, anterior, posterior or between cruciate ligaments [7]. MR is the gold standard for detecting GC1. Recently observation by ultrasonography is considered useful for identifying and locating the lesion, as well as being a conservative approach to treat cystic lesions [1].

\section{Case Report}

A 17-year-old Caucasian female, a soccer player, presented with a 9 month history of left knee pain, mainly in the posterior and medial aspects of the knee, combined with slightly back swelling. Pain was exacerbated with exercise, especially squatting, and partially alleviated with rest. She had no history of a knee major traumatic event (Figure 1). She complained of knee pain on soccer playing, one of the main reasons to suspend this practice. She alsorefers no confidence on demanding tasks.

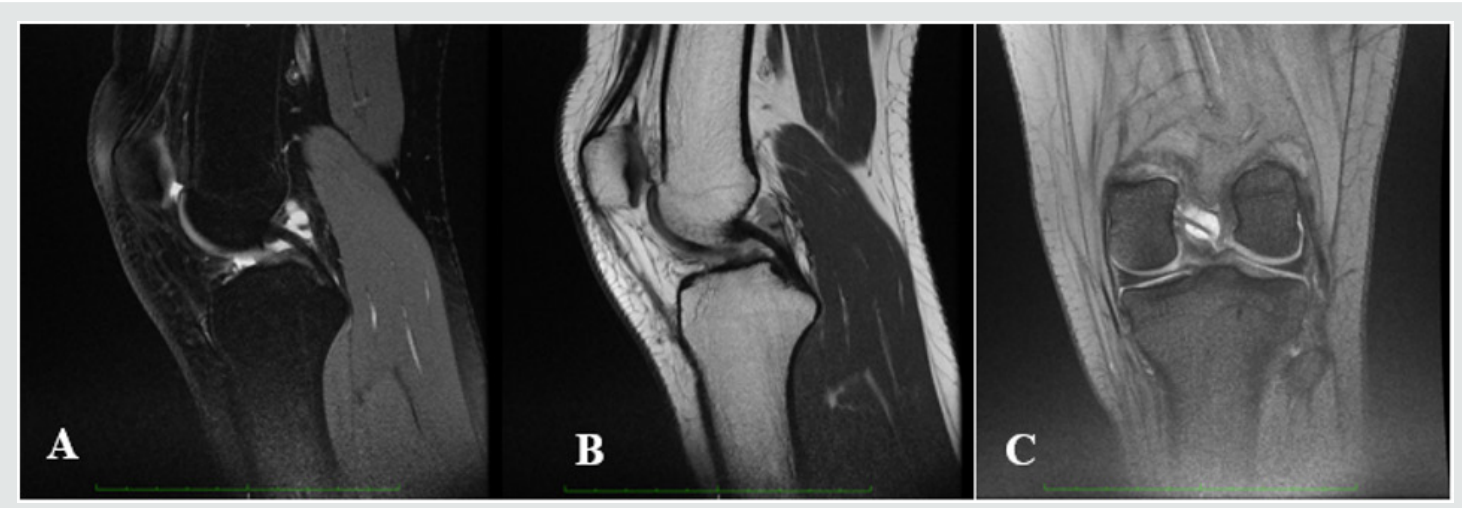

Figure 1: Knee MRI of PCL GC; A: T2 sagittal view FS; B: T2 sagittal view FSE; C: T2* coronal view.

In clinical examination we identified a light quadriceps atrophy, compared to the opposite side, and symmetry in active range of motion (ROM). Passive ROM was limited (10 degrees) and painful in extreme flexion and symmetric in hyperextension. Meniscal tests and varus-valgus stress tests were negative. Some tenderness on palpation of the popliteal aspect but no pain or even tenderness 
was verified in medial or lateral joint line. Plain radiographs were normal. A knee magnetic resonance image (MRI) was then performed regarding the potential diagnosis of a joint cyst so it was acquired weighted T2 sagittal sequences with and without fat suppression and T2* coronal. The MRI revealed a high-signal well-defined, ovoid shaped formation extending along PCL on both T2-weighted images and fat-suppression sequences, measuring $18 \mathrm{mmm}$ length compatible with a PCL cyst. Considering that in the last consultation there is no pain or appreciable impairment of mobility, and also the athlete does not intend to continue the practice of soccer, the choice was made for conservative treatment. Proprioceptive closed-chain training and quadriceps and hamstrings muscle strengthening were prescribed for 3 months, 3 $\mathrm{x}$ week. Currently she runs $5 \mathrm{x}$ week ( $40 \mathrm{~min}$ per day) without pain and functional disability. A new appointment will be made in six months.

\section{Discussion}

Etiology and pathogenesis of PCLGC are unclear, however it is proposed that repetitive microtrauma of joint and soft tissue can promote expansion of mucin from ligament fibers and acting as a potential trigger [20]. Recognition of PCLGC as a clinical entity leading knee pain and impairment is increasing due to the sensitivity of MRI to identify intra-articular abnormalities. The typical finding is an ovoid fluid filled cystic lesion which can frequently be multilocular in the intercondylar notch of the knee $[22,25]$. In our case report MRI shows a cystic multilocular mass with fluid signal intensity within the synovial layer of the PCL. Although most knee cysts are asymptomatic, in some case they could be a relevant source of pain [20,21]. Clinical manifestations of a knee cyst are mostly dependent on the pathologic process involved, along with its location, size, mass effect, and relationship to surrounding structures [26]. The typical presentation of symptomatic PCLGC include posterior knee pain, restriction of ROM, stiffness and mild swelling [20,21].

Limited ROM is a typical finding with an intra-articular ganglion arising from the PCL, mainly with inability and pain to extreme flexion due to the compression of the cyst mass between the PCL and the posterior joint capsule. With this clinical picture in mind, athletes between 20 and 40 years old who present knee pain with restriction on hyperextension or full flexion, with no previous macrotraumatic report or knee instability, should raise a high level of suspicion for intra-articular ganglion cysts. Only symptomatic PCLGC need to undergo treatment. There a broad spectrum of treatments described for these lesions, from a rehabilitation program focused on ROM, strengthening and proprioception to avoid kinetic impairment, to ultrasound or CT-guided aspiration or infiltration, or even arthroscopic excision. Treatment choice must take into account several criteria such as level of activity, time for recovery, risk of joint damage and recurrence of the cyst. Arthroscopic treatment has demonstrated good outcomes with up to $95 \%$ of patients reporting good results and associated with the lowest recurrence rate, but it needs an hospitalization, anesthesia and a longer recovery period, which can become a major problem when we are dealing with competitive sports $[23,24]$.
Athletes require quick return to play with minimal side effects, so we need to take into account less invasive treatments like US or CT-guided procedures, or even load management in addition to a rehabilitation program.

\section{Conclusion}

CCP is a rare and often asymptomatic condition. Its pathogenesis and prognosis are still unclear. In a young adult with posterior knee pain (popliteal aspect), no history of major event, limited ROM (hyperextension and extreme flexion), meniscus and ligament test negative and no confidence in demanding tasks it is important to think about this condition. The therapeutic option stems from the patient's characteristics, but US or CT- guided puncture should be considered.

\section{References}

1. Vilella G, Guerrisi P, Lucignani G, Pasquali G, Drudi FM (2014) Ultrasoundguided aspiration and steroid injection of a posterior cruciate ligament ganglion cyst: report of a case. J Ultrasound 18(3): 283-286.

2. Derman P, Kamath AF, Kelly Iv JD (2011) Ganglion cysts of the posterior cruciate ligament. Am J Orthop 40(5): 257-258.

3. Kodaira S, Nakajima T, Takahashi R, Moriya S, Nakagawa T, et al. (2016) A case of intra-articular ganglion cysts of the knee joint: correlation between arthroscopic and magnetic resonance imaging. BMC Med Imaging 16(1): 36.

4. Tie K, Wang H, Zhao X, Tan Y, Qin J, et al. (2018) Clinical manifestation and arthroscopic treatment of symptomatic posterior cruciate ligament cyst. J Orthop Surg Res 13(1): 84.

5. Andreozzi V, Monaco E, Conteduca F, Iorio R, Mazza D, et al. (2019) Diagnosis and Treatment of a Symptomatic Posterior Cruciate Ganglion Cyst in a Child with Autism. Case Rep Orthop 2019: 9192347.

6. Kim RS, Kim KT, Lee JY, Lee KY (2003) Ganglion cysts of the posterior cruciate ligament. Arthroscopy 19(6): E36-E40.

7. Stein D, Cantlon M, MacKay B, Hoelscher C (2013) Cysts about the knee: evaluation and management. J Am Acad Orthop Surg 21: 469-479.

8. McCarthy CL, McNally EG (2004) The MRI appearance of cystic lesions around the knee. Skeletal Radiol 33(4): 187-209.

9. Kim MG, Kim BH, Choi JA, et al. (2001) Intraarticular ganglion cysts of the knee: Clinical and MR imaging features. Eur Radiol 11(5): 834-840.

10. Joo Y, Kim Y (2012) Symptomatic posterior cruciate ganglion cyst causing impingement between posterior root of the medial meniscus and anterior to the posterior cruciate ligament. Knee Surgery Related Research; 24(1): 52-55.

11. Krudwig W, Shulte K, Heinemann C (2004) Intra-articular ganglyon cysts of the knee joint: a report of 85 cases and review of the literature. Knee Surg Sports Traumatol Arthrosc 12(2): 123-129.

12. Tie K, Wang H, Zhao X, Tan Y, Quin J, et al. (2018) Clinical manifestation and arthroscopic treatment of symptomatic posterior cruciate ligament cyst. Journal of Orthopaedic Surgery and Research 13(1): 84.

13. Mao Y, Dong Q Wang Y (2012) Ganglion Cysts of the cruciate ligaments: a series of 31 cases and review of the literature. BMC Musculoskeletal Disorders 13(1): 137.

14. Bui-Mansfield LT, Youngberg RA (1997) Intraarticular ganglia of the knee: Prevalence, presentation, etiology, and management 168(1): 123127.

15. Kang CN, Kim DW, Kim DJ, Kim SJ (1999) Intra-articular ganglion cysts of the knee. Arthroscopy 15(4): 373-378. 
16. Shetty G, Nha K, Chae D, Kang K, Yoon J, et al. (2008) Knee 15: 325-329.

17. Lunhao B, Yu S, Jiashi W (2011) Diagnosis and treatment of ganglion cysts of the cruciate ligaments. Arch Orthop Trauma Surg 131(8): 10531057.

18. Fritschy D, Fasel J, Imbert J, Bianchi S, Wirth C (2006) The popliteal cyst. Knee Surg Sports Traumatol Arthrosc 14(7): 623-628.

19. Cristina A, Wilson P (1964) Popliteal cysts in adults and children: A review of 90 cases. Arch Surg 88(3): 357-363.

20. Bui-Mansfield LT, Youngberg RA (1997) Intraarticular ganglia of the knee: prevalence, presentation, etiology, and management. 168(1): 123-127.

21. Lunhao B, Yu S, Jiashi W (2011) Diagnosis and treatment of ganglion cysts of the cruciate ligaments. Arch Orthop Trauma Surg 131(8): 10531057.
22. Marra MD, Crema MD, Chung M (2008) MRI features of cystic lesions around the knee. Knee 15(6): 423-438.

23. DeFriend DE, Schranz PJ, Silver DA (2001) Ultrasound-guided aspiration of posterior cruciate ligament ganglion cysts. Skeletal Radiol 30(7): 411414 .

24. Krudwig WK, Schulte KK, Heinemann C (2004) Intra-articular ganglion cysts of the knee joint: a report of 85 cases and review of the literature. Knee Surg Sports Traumatol Arthrosc 12(2): 123-129.

25. Coakley FV, Finlay DB, Harding ML, Oni OA (1996) The nature and significance of cysts found at MR imaging behind the posterior cruciate ligament of the knee. The Knee 3(3): 139-143.

26. Beall DP, Ly JQ, Wolff JD, Sweet CF, Kirby AB, et al. (2005) Cystic masses of the knee: magnetic resonance imaging findings. Curr Probl Diagn Radiol 34(4): 143-159.

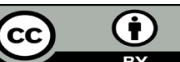

This work is licensed under Creative Commons Attribution 4.0 License

To Submit Your Article Click Here: Submit Article

DOI: $10.32474 /$ OSMOAJ.2019.03.000158

$\begin{gathered}\text { Orthopedics and Sports Medicine } \\ \text { Open Access Journal }\end{gathered}$
Assets of Publishing with us
- Global archiving of articles
- Immediate, unrestricted online access
- Rigorous Peer Review Process
- Authors Retain Copyrights
- Unique DoI for all articles

